\title{
GLYCOGEN STORAGE DISEASE TYPE IB: A DEFECT IN THE GLUCOSE-6-PHOSPHATE TRANSPORT SYSTEM IN MICROSOMAL MEMBRANE
}

\author{
(The Japan Society of Human Genetics Award Lecture)
}

\author{
Keiya TADA \\ Department of Pediatrics, Tohoku University School of Medicine, \\ Sendai 980, Japan
}

Glycogen storage disease (GSD) type 1, von Gierke disease, is well known as the disorder, in which an enzyme defect was first demonstrated in 1952 by Cori and Cori, among many inborn errors of metabolism. However, with the accumulation of cases, there have been the reports of the patients with von Gierke disease who showed normal activity of glucose-6-phosphatase (G6Pase) in the liver. Now GSD type 1 has been divided into two subtypes (Auerbach and DiGeorge, 1975). Type Ia is due to a defect in G6Pase and type Ib is clinically indistinguishable from type Ia but shows normal activity of hepatic G6Pase. The defect in type Ib had been unknown until we demonstrated that glucose-6-phosphate (G6P) transport system in microsomal membrane is defective (Narisawa et al., 1978; Igarashi et al., 1979).

This paper describes a series of our study on GSD type Ib.

\section{High latency of G6Pase in intact microsomes}

The patient Y.S. was born in 1975, after an uneventful pregnancy and full term delivery. Her birth weight was $2,850 \mathrm{~g}$. Her parents were healthy and unrelated. There were no siblings. On 15th day of age she became irritable, followed by convulsions, when a marked hypoglycemia (below $20 \mathrm{mg} / 100 \mathrm{ml}$ ) was noticed. An intravenous infusion of glucose resulted in a recovery from clinical symptoms. Thereafter, she showed frequent episodes of hypoglycemia at fasting and consequently growth was retarded.

At the age of 10 months, the patient was referred to our University Hospital for examinations. On admission, her weight was $7.6 \mathrm{~kg}$ and length $64.7 \mathrm{~cm}$ (normal, $8.9 \mathrm{~kg}$ and $71.8 \mathrm{~cm}$, respectively). The liver edge was palpated $10 \mathrm{~cm}$ below the right costal margin. She showed doll-like appearance.

The laboratory findings are summarized in Table 1. Thus the patient Y.S. showed the typical features of GSD Type I, such as hepatomegaly, growth retardation, doll-like appearance, rapid development of hypoglycemia after only $2 \mathrm{hr}$ fast-

Presented on the occasion of receiving the Japan Society of Human Genetics Award at the annual meeting of the Japan Society of Human Genetics, Takarazuka, Japan, November 9-11, 1983. 
Table 1. Laboratory findings of patient Y.S.

\begin{tabular}{lrc}
\hline Blood sugar & Below $25 \mathrm{mg} / 100 \mathrm{ml}$ & $\downarrow$ \\
Blood lactate & $48 \mathrm{mg} / 100 \mathrm{ml}$ & $\uparrow$ \\
Serum triglycerides & $940 \mathrm{mg} / 100 \mathrm{ml}$ & $\uparrow$ \\
Serum cholesterol & $245 \mathrm{mg} / 100 \mathrm{ml}$ & $\uparrow$ \\
Serum uric acid & $10.6 \mathrm{mg} / 100 \mathrm{ml}$ & $\uparrow$ \\
\hline
\end{tabular}

Glucagon test: no response of blood glucose. Galactose loading test: no rise of blood glucose. Fructose loading test: no rise of blood glucose.

ing, hyperlipidemia, hyperlactic acidemia and hyperuric acidemia. There was no rise of blood glucose in response to administered glucagon, galactose or fructose. Liver biopsy demonstrated an accumulation of glycogen ( $8.5 \%$ of wet weight) with normal structure. Enzyme analysis of the liver specimen, stored at $-25^{\circ} \mathrm{C}$ for a day after its collection, revealed normal activity of G6Pase $\left(4.5 \mu \mathrm{mol} \mathrm{P}_{\mathrm{i}}\right.$ formed $/ \mathrm{min} / \mathrm{g}$ liver; normal range 3.3-5.9), and elevated activity of fructose-1,6-diphosphatase activity $(19.9 \mu \mathrm{mol} \mathrm{NADPH}$ generated/min/g liver; normal range 5.7-10.0).

The results of in vivo tests were all consistent with a defect in hepatic G6Pase (Fig. 1). But the activity of G6Pase was normal in the liver specimen stored in freezing. Therefore, the patient was diagnosed as GSD Type Ib.

Arion et al. (1975) postulated that G6Pase activity results from the coupling of two components of the microsomal membrane: 1) a glucose-6-phosphate-specific transport system which functions to shuttle G6P from the cytoplasm to the lumen of endoplasmic reticulum; and 2) a catalytic component, glucose-6-phosphate phosphohydrolase, bound to the luminal surface of the membrane.

Under such circumstances, a possibility remains that glycogen storage disease of Type $\mathrm{Ib}$ could result from a defect in the G6P transport system in the function of microsomal G6Pase.

At the age of 3 years, a surgical operation of mesentericocaval shunt was performed, because she had repeated episodes of severe hypoglycemia, metabolic acidosis and recurrent infections requiring frequent hospitalizations.

The following studies were performed, with use of liver specimen obtained in the fasting state at the operation.

Liver specimens immediately after biopsied were homogenized with $0.25 \mathrm{M}$ sucrose solution and centrifuged to remove nuclei and cellular debris. The supernatants were divided in two portions: one is treated with deoxycholate for two hours in cold to destroy microsomal membrane and the other is supplemented with distilled water as described by Nordie and Arion (1965). Then G6P phosphohydrolase activities were measured both in intact microsomes and disrupted microsomes according to the procedure of Nordie and Arion (1966).

As shown in Table 2, the activity in patients with GSD Type Ia and Ib was found to be markedly decreased as compared with those in control subjects, when 


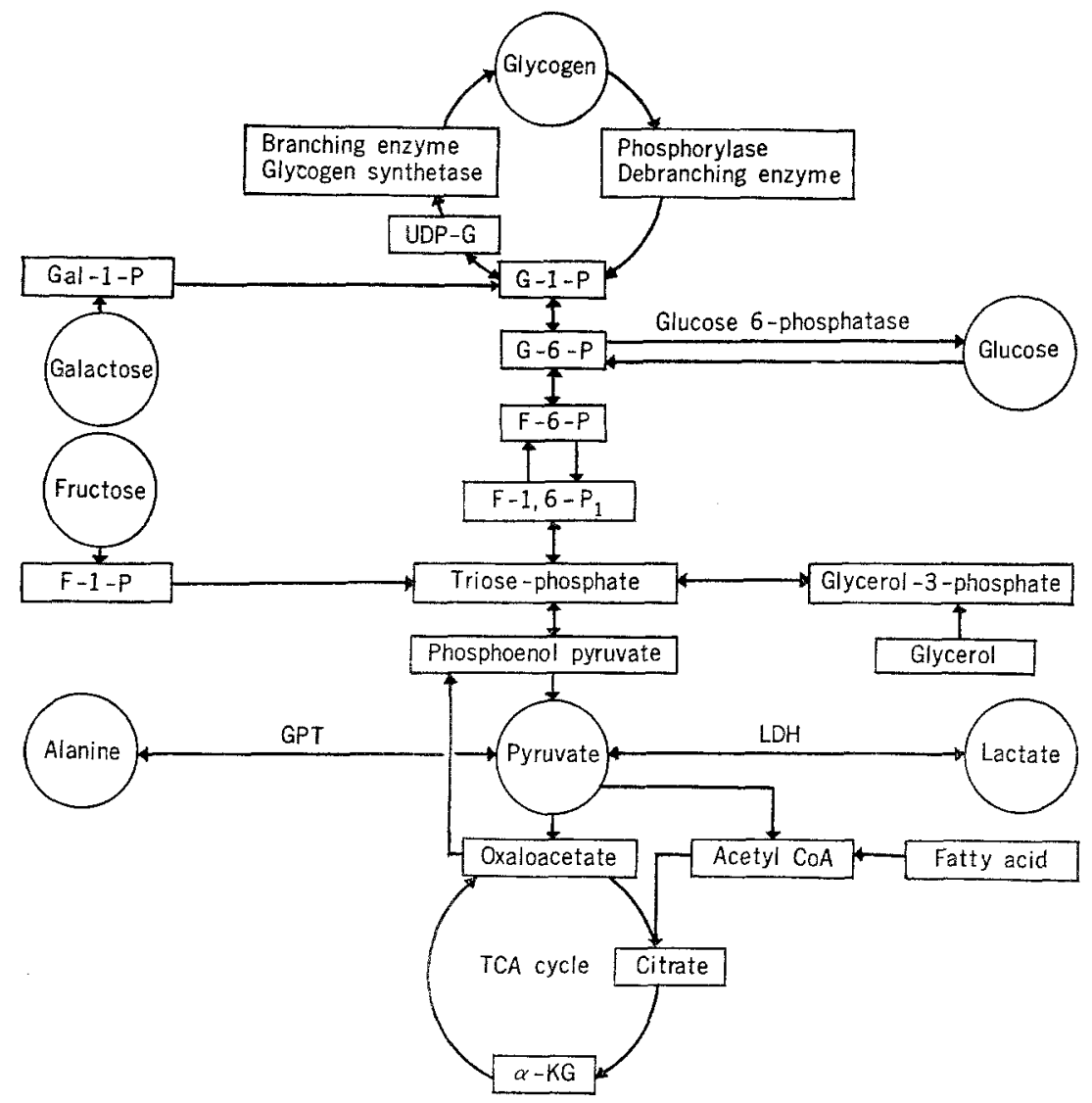

Fig. 1. Glycogen metabolism.

Table 2. Glucose-6-phosphatase activity of livets from patient Y.S., the patients with glycogen storage disease Type Ia, and control subjects.

\begin{tabular}{lccc}
\hline & $\begin{array}{c}\text { Intact microsomes } \\
\left(\mu \mathrm{mol} \mathbf{P}_{\mathrm{i}} \text { formed/min/g liver) }\right.\end{array}$ & $\begin{array}{c}\text { Disrupted microsomes } \\
(\%)\end{array}$ \\
\hline Patient Y.S. (3 years) & 0.8 & 10.2 & $\begin{array}{c}\text { Latency a } \\
(\%)\end{array}$ \\
\hline Control subjects & & & 92.3 \\
\hline (52 y) & 3.6 & 7.5 & 52.0 \\
$2(71 \mathrm{y})$ & 2.0 & 4.4 & 54.5 \\
$3(10 \mathrm{y})$ & 3.3 & 4.5 & 26.7 \\
$4(1 \mathrm{y})$ & 4.8 & 6.2 & 22.6 \\
$5(2 \mathrm{y})$ & 5.9 & - & - \\
$6(3 \mathrm{y})$ & 4.8 & - & \\
\hline Cases of glycogen storage disease Type Ia & 0.61 & 0.45 & 44.4 \\
$1(25 \mathrm{y})$ & 0.20 & 0.36 & \\
$2(27 \mathrm{y})$ & & & \\
\hline
\end{tabular}

a Latency is defined as $\left(1-\frac{\text { activity in intact microsomes }}{\text { activity in disrupted microsomes }}\right) \times 100$

Vol. 29, No. 2, 1984 
the assays were made in the absence of deoxycholate. However, the treatment of liver homogenates with detergent produced a restoration of the activity up to normal in the patient Y.S. On the other hand, no restoration of the activity by the detergent treatment was observed in the patients with Type Ia of GSD.

A high latency of G6Pase observed in intact microsomes of the patient Y.S. can be explained by a lack of the G6P transport system, because the treatment with detergent brought the restoration of G6Pase activity. Recently much evidence has been accumulating for the existence of G6P transport system in the endoplasmic reticulum of rat liver. Our observation in this particular patient Y.S. supports an existence of the G6P transport system in microsomes of human liver.

Our earlier observation, that the activity of hepatic G6Pase in the patient Y.S. was normal, can be explained by a decrease in the latency of the enzyme during the storage at $-25^{\circ} \mathrm{C}$ which may destroy microsomal membranes.

In fact, we investigated the effect of storage of liver tissue for $24 \mathrm{hr}$ at $-25^{\circ} \mathrm{C}$ on the activity of G6Pase in patient Y.S. A significant decrease of latency of the activity of G6Pase was observed during the storage, as shown in Table 3. Furthermore, there was observed an agreement in the decrease of latency during the storage between G6Pase of the patient and mannose-6-phosphate phosphohydrolase of rat (Fig. 2). This indicates that G6Pase activity is manifested by a partial destruction of microsomal membrane and that the G6P transport system in the membrane is defective in the patient because the transport system of mannose-6phosphate (M6P) does not exist in microsomal membrane of rat (Arion et al., 1980). Usually the assay of the enzyme used to be made after the storage in freezing. This is the reason why the patients with Type Ib were reported to show normal activity of G6Pase.

Our finding mentioned above were subsequently confirmed by other investigators (Beaudet et al., 1980; Lange et al., 1980; Corbeel et al., 1981; Schaub et al., 1981). And it is now established that primary lesion of glycogen storage disease

Table 3. Effects of storage of liver tissues for $24 \mathrm{hr}$ at $-25^{\circ} \mathrm{C}$ on the activities of glucose-6-phosphatase in patient Y.S. and of M6P phosphohydrolase in rats.

\begin{tabular}{lccc}
\hline \multicolumn{4}{c}{ Glucose-6-phosphatase activity in patient Y.S. } \\
\hline & $\begin{array}{c}\text { Without deoxycholate } \\
\text { treatment } \\
(\mu \mathrm{mol} / \mathrm{min} / \mathrm{g} \text { liver })\end{array}$ & $\begin{array}{c}\text { With deoxycholate } \\
\text { treatment }\end{array}$ & $\begin{array}{c}\text { Latency } \\
(\%)\end{array}$ \\
\hline Fresh liver & 0.9 & 12.8 & 93 \\
Stored liver & 3.4 & 12.4 & 73 \\
\hline & Mannose-6-phosphohydrolase activity in rats $(n=7, \mathrm{M} \pm \mathrm{SD})$ & \\
\hline Fresh liver & $1.06 \pm 0.20$ & $9.85 \pm 2.61$ & $89.1 \pm 0.97$ \\
Stored liver & $2.83 \pm 1.48$ & $10.67 \pm 3.11$ & $74.6 \pm 7.5$ \\
\hline
\end{tabular}




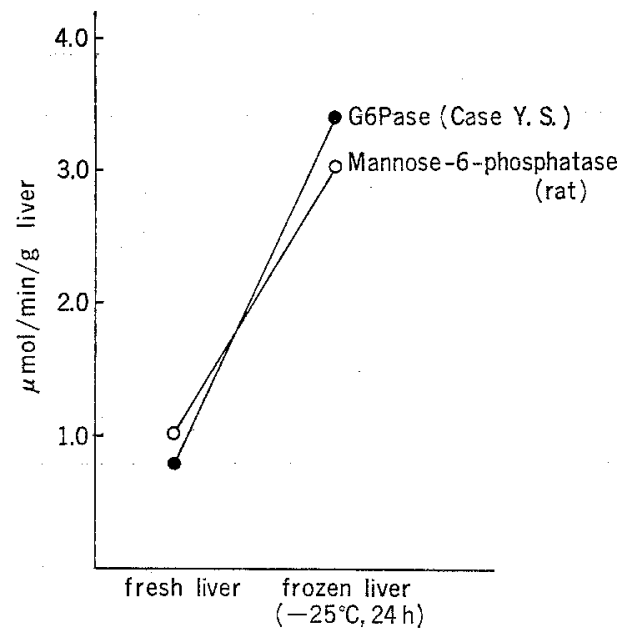

Fig. 2.

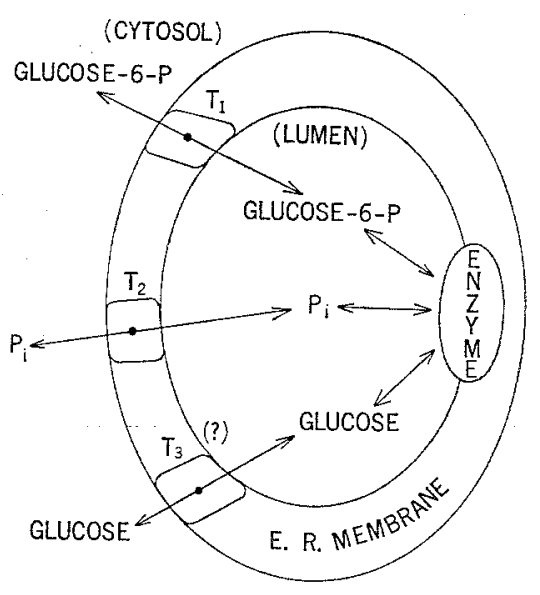

Fig. 3.

Fig. 2. Effect of storage of liver tissues for $24 \mathrm{hr}$ at $-25^{\circ} \mathrm{C}$ on the activities of G6Pase in patient Y.S. and of M6Pase in rats.

Fig. 3. Schematization of glucose-6-phosphatase system of the hepatic endoplasmic reticulum (Arion et al., 1980). $T_{1}$ : transport system for G6P. $T_{2}$ : transport system for pyrophosphate. $T_{3}$ : transport system for glucose.

Type $\mathrm{Ib}$ lies in the G6P transport system (G6P translocase) in microsomal membrane.

\section{A specific defect in G6P transport system}

G6Pase is known to be a multifunctional enzyme, that is, not only G6P but also M6P and pyrophosphate act as the substrate. According to Arion et al. (1980), there may be separate transport systems for G6P and pyrophosphate in microsomal membrane but no transport system for M6P exists (Fig. 3).

In order to confirm this assumption, we investigated the activities of pyrophosphatase and mannose-6-phosphatase (M6Pase) both in intact microsomes and disrupted microsomes in the livers from the patients with GSD Type Ia and Ib.

In Type $\mathrm{Ib}$, pyrophosphatase activity was normal in both intact and disrupted microsomes fractions whereas in Type Ia the activity was very low in both fractions (Table 4). These findings indicate that there are separate transport systems for G6P and pyrophosphate and Type $\mathrm{Ib}$ is due to a specific defect in G6P transport system.

There was no significant difference in latency of M6Pase between Type Ib and controls (Table 5). Type Ia shows very low activity both in intact and disrupted microsomes. The reason why the latency of M6Pase is not $100 \%$, may be due to a partial disruption of microsomal membrane during the homogenation. In other words, a decrease in the latency of M6Pase is thought to be an index of microsomal integrity. Therefore, when we corrected the latency of G6Pase by that of 
Table 4. Pyrophosphate phosphohydrolase activities $\left(\mu \mathrm{mol} \mathrm{P}_{\mathbf{i}}\right.$ per min per $\mathrm{g}$ liver) in the liver.

\begin{tabular}{lccc}
\hline & Intact microsomes & Disrupted microsomes & Latency $(\%)$ \\
\hline GSD-Ib & & & \\
Patient Y.S. & 6.07 & 19.06 & 68.1 \\
Patient Y.M. & 5.37 & 14.37 & 62.7 \\
GSD-Ia (5) & $0.04 \pm 0.03$ & $0.08 \pm 0.06$ & 0 \\
Controls (5) & & & \\
Mean \pm SD & $3.23 \pm 0.77$ & $9.79 \pm 3.78$ & $64.4 \pm 10.6$ \\
Range & $2.33-4.04$ & $5.75-13.71$ & $47.8-74.4$ \\
\hline
\end{tabular}

Table 5. M6Pase in liver ( $\mu \mathrm{mol} \mathrm{P} / \mathrm{min} / \mathrm{g})$.

\begin{tabular}{rccc}
\hline & Intact microsomes & Disrupted microsomes & Latency $(\%)$ \\
\hline GSD-Ib & & & \\
YU. S. & 0.47 & 11.91 & 96.1 \\
MORI. & 0.70 & 8.71 & 92.0 \\
GSD-Ia & 0.02 & 0.09 & - \\
& 0.06 & 0.21 & - \\
& 0.11 & 0.14 & - \\
& 0.08 & 0.16 & - \\
Controls & 0.07 & 0.03 & - \\
& 1.09 & 7.08 & 84.6 \\
& 0.49 & 3.62 & 86.5 \\
& 1.55 & 7.37 & 79.0 \\
\hline
\end{tabular}

M6Pase, the patient Y.S. and Y.T. (Type Ib, infantile cases) showed almost $100 \%$ of latency, indicating the absence of G6P transport system (Table 6).

3. Genetic heterogeneity in glycogen storage disease Type Ib

Among Type Ib patients, Y.M. is an adult case which showed $90 \%$ of latency indicating residual activity of G6P translocase (Table 6).

Patient Y.M. is a 25-year-old woman. Hepatomegaly was first noticed at 4 years of age, but there was no clinical symptom. She was diagnosed GSD Type Ib at the age of 20 years. She had short stature and hepatomegaly. Although she showed subnormal levels of blood glucose on fasting, she did not present clinical evidence of hypoglycemia. A mild elevation of triglycerides, lactate and uric acid and a poor response of blood glucose to glucagon were found. She had neither neutropenia nor episodes of recurrent infections.

In patient Y.M. the kinetics of G6P translocase were assayed on intact microsomes. The apparent $K_{\mathrm{m}}$ value was found to be about 16 -fold higher than that 
Table 6. Latency of G6Pase and pyrophosphatase corrected by the latency of M6Pase.

\begin{tabular}{lccc}
\hline & $\begin{array}{c}\text { Intact } \\
\text { microsomes }\end{array}$ & $\begin{array}{c}\text { Disrupted } \\
\text { microsomes }\end{array}$ & Latency (\%) \\
\hline G6P phosphohydrolase & & & \\
GSD-Ib & 0.003 & 12.60 & 100.0 \\
Patient Y.S. & 0.02 & 5.68 & 99.6 \\
Patient Y.T. & 0.85 & 9.18 & 90.7 \\
Patient Y.M. & $0.09 \pm 0.04$ & $0.12 \pm 0.04$ & 0 \\
GSD-Ia (6) & $4.25 \pm 0.80$ & $6.26 \pm 1.22$ & $31.7 \pm 6.6$ \\
Controls (6) & & & 68.1 \\
Pyrophosphate phosphohydrolase & & 19.08 & 68.3 \\
GSD-Ib & 6.07 & 7.10 & 62.7 \\
Patient Y.S. & 2.25 & 14.37 & 0 \\
Patient Y.T. & 5.73 & $0.08 \pm 0.06$ & $64.4 \pm 10.6$ \\
Patient Y.M. & $0.04 \pm 0.03$ & $9.79 \pm 3.78$ & \\
GSD-Ia (5) & $3.12 \pm 0.77$ & & \\
Controls (5) & & & \\
\hline
\end{tabular}

a Calculated using the formula of Arion et al. (1980).

of the controls (Narisawa et al., 1983; Tada et al., 1983). This suggests that the residual activity of G6P translocase in patient Y.M. is caused by a structural gene mutation.

On the basis of the above findings, it may be assumed that the clinical severity of Type Ib depends on the level of the residual activity of the G6P translocase.

Patients with GSD Type Ia and Type Ib show similar clinical features, but a difference exists in that the patients with GSD Type Ib have neutropenia and episodes of recurrent infections (Beaudet et al., 1980; Bartram et al., 1981; Narisawa et al., 1981). Our patients with infantile cases of Type Ib, who showed almost complete absence of G6P translocase, had episodes of recurrent infections and neutropenia but an adult patient of Type $\mathrm{Ib}$, who showed a residual activity of G6P translocase, had neither episodes of recurrent infections nor neutropenia. These findings suggest that a residual activity of G6P translocase may be related to the absence of neutropenia, although the mechanism is unclear.

\section{A direct evidence for the defect in G6P transport system}

So far, the analysis of G6P translocase has been made indirectly by comparing the G6Pase activity in intact microsomes and in disrupted microsomes.

Recently we developed a method to investigate the uptake of G6P by hepatic microsomes (Igarashi et al., 1984). Microsomes were isolated from fresh liver according to the procedure of Kamath et al. (1971) (Fig. 4). The isolated microsomes were immediately put on membrane filter (pore size $0.8 \mu \mathrm{m}$ ) and the incu- 
$20 \%$ fresh homogenate with solution (A)

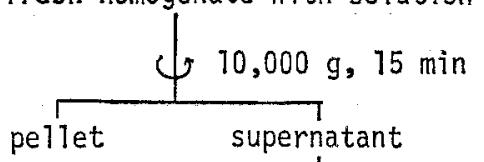

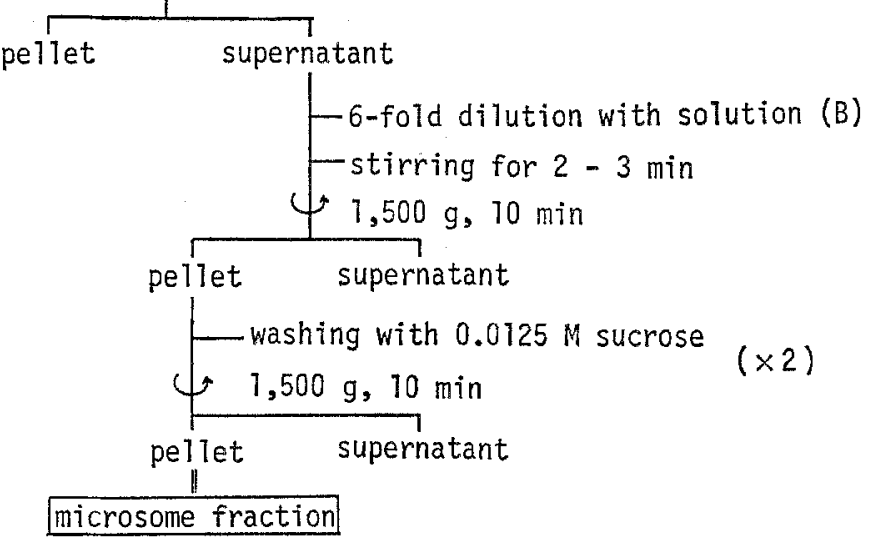

Solution [A]: $0.25 \mathrm{M}$ sucrose, $50 \mathrm{~mm}$ Tris-Cl buffer pH 7.5, $25 \mathrm{~mm} \mathrm{KCl}, 5 \mathrm{~mm}$

$\mathrm{MgCl}_{2}, 8 \mathrm{mM} \mathrm{CaCl}_{2}$.

Solution [B]: $0.0125 \mathrm{M}$ sucrose, $5 \mathrm{~mm} \mathrm{MgCl} 2,8 \mathrm{~mm} \mathrm{CaCl}$. \# All operations are carried out between $0-4^{\circ} \mathrm{C}$.

Fig. 4. Preparation of hepatic microsome fraction.

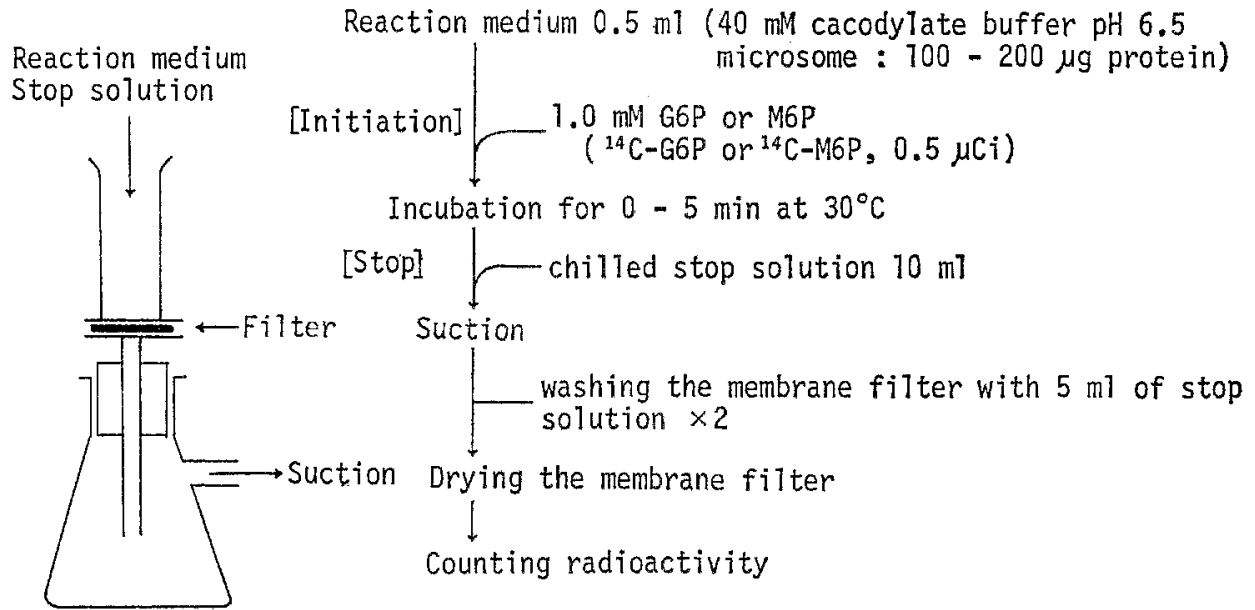

Stop solution: 0.25 м sucrose, 50mm Tris buffer pH 7.5, $25 \mathrm{~mm} \mathrm{KCl,} 5 \mathrm{~mm} \mathrm{MgCl} 2,8 \mathrm{~mm} \mathrm{CaCl}$.

Fig. 5. Assay method for uptake of G6P into microsomes.

bation with a tracer of $\left[{ }^{14} \mathrm{C}\right] \mathrm{G} 6 \mathrm{P}$ or $\left[{ }^{14} \mathrm{C}\right] \mathrm{M} 6 \mathrm{P}$ was done (Fig. 5). Incubation medium (final volume $0.5 \mathrm{ml}$ ) contained $40 \mathrm{~mm}$ cacodylate buffer, $\mathrm{pH} 6.5,1 \mathrm{~mm}$ G6P or M6P and microsomes (about $200 \mu \mathrm{g}$ of protein). As a tracer, $0.5 \mu \mathrm{Ci}$ of $\left[{ }^{14} \mathrm{C}\right] \mathrm{G} 6 \mathrm{P}$ (NEN, Boston) or $\left[{ }^{14} \mathrm{C}\right] \mathrm{M} 6 \mathrm{P}$ (NEN, Boston, specially ordred) was used, respectively. 
The incubation mixture was kept at $30^{\circ} \mathrm{C}$. The reaction was stopped by diluting with 20 -fold excess of cold $0.25 \mathrm{M}$ sucrose solution supplemented with $50 \mathrm{~mm}$ Tris- $\mathrm{HCl}$ buffer ( $\mathrm{pH} 7.5), 25 \mathrm{mM} \mathrm{KCl}, 5 \mathrm{mM} \mathrm{MgCl}{ }_{2}$ and $8 \mathrm{mM} \mathrm{CaCl}_{2}$ at any time from 0 to $5 \mathrm{~min}$. The microsomes were immediately collected on a washed membrane filter (TOYO ROSHI Co., Ltd., Type TM-80P $24 \mathrm{~mm}$ in diameter, Tokyo) and washed twice with $5 \mathrm{ml}$ of the above sucrose solutions. The filter membrane was dried at room temperature. The radioactivity on filter membrane was counted by liquid scintillation counter (Intertec, SL30). All experiments were made duplicated. The uptake at each time was estimated after subtracting the count of 0 time from that of each time and expressed nmol/mg protein.

Uptake of G6P and M6P by microsomes of rat hepatocyte was shown in Fig. 6. When $1 \mathrm{mM}$ of G6P was added to the incubation mixture, the uptake was rapidly and linearly increased until $90 \mathrm{sec}$. Then it reacted to a plateau. On the other hand, the uptake of M6P was almost negligible at any time from 1 min to 5 min. The results on human hepatic microsomes were shown in Fig. 7. A significant uptake of G6P by microsomes was observed in patients with GSD Ia and controls, although the uptake in patients with GSD Ia was lower than that in controls. On the contrary, the patient with GSD Ib showed negligible uptake of GóP. Uptake of $\mathrm{M} 6 \mathrm{P}$ in controls was undetectable (data not shown). These findings provide the direct evidence that G6P specific transport system exists in human microsomal membrane and GSD Ib is due to a defect of the G6P transport system.

The reason why the uptake of G6P in GSD Ia was lower than that of controls may be due to a phenomenon that microsomes in GSD Ia are already filled with G6P because of a defect in G6Pase. Indeed, in preliminary experiment, the content of G6P in liver tissue from patients with GSD Ia was found to be high as compared with that of controls.

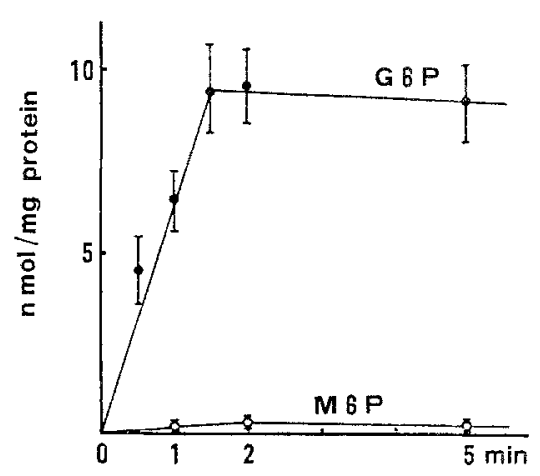

Fig. 6.

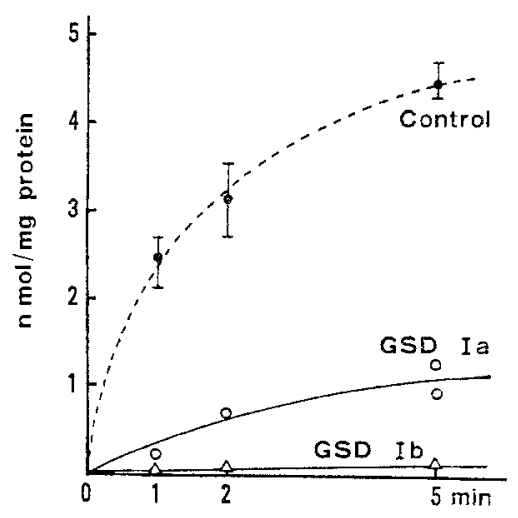

Fig. 7.

Fig. 6. Uptake of G6P and M6P by microsomes of rat hepatocyte.

Fig. 7. Uptake of G6P by hepatic microsomes from the patients with GSD type Ia and Ib. 


\section{CONCLUSION}

A series of our study revealed that the primary lesion of GSD Type Ib exists in the G6P transport system in microsomal membrane. A distinct evidence of the existence of a specific transport system of G6P in microsomal membrane was obtained through these studies. This is the first example of a genetic disorder involving the transport system of intracellular membrane.

Acknowledgements This work was supported by grants from the Ministry of Education, Science and Culture and the Ministry of Health and Public Welfare, Japan.

\section{REFERENCES}

Arion, W.J., Lange, A.J., Walls, H.E., and Ballas, L.M. 1980. Evidence for the participation of independent translocase for phosphate and glucose-6-phosphate in the microsomal glucose-6phosphatase system. J. Biol. Chem. 255: 10396-10406.

Arion, W.J., Wallin, B.K., Lange, A.J., and Ballas, L.M. 1975. On the involvement of a glucose6-phosphate transport system in the function of microsomal glucose-6-phosphatase. Mol. Cell Biochem. 67: 75-83.

Auerbach, V.H. and DiGeorge, A.M. 1975. Glycogenosis: Disorders of glycogen metabolism. In Nelson Textbook of Pediatrics, Vaughan, V.C. and McKay, R.J., eds., 10th ed., W.B. Saunders Company, Philadelphia, pp. 439-442.

Bartram, C.R., Przyrembel, H., Wendel, U., Bremer, H.J., Schaub, J., and Haas, J.R. 1981. Glycogenosis type Ib complicated by severe granulocytopenia resembling inherited neutropenia. Eur. J. Pediatr. 137: 81-88.

Beaudet, A.L., Anderson, D.C., Michels, V.V., Arion, W.J., and Lange, A.J. 1980. Neutropenia and imparied neutrophil migration in type $\mathrm{Ib}$ glycogen storage disease. J. Pediatr. 97: 906910.

Corbeel, L., Hue, L., Lederer, B., Barsy, T.D., Berthe, V.D., Delvlieger, H., Jacken, J., Brack, P., and Eckels, R. 1981. Clinical and biochemical findings before and after portacaval shunt in a girl with type Ib glycogen storage disease. Pediatr. Res. 15: 58-61.

Igarashi, Y., Kato, S., Narisawa, K., and Tada, K. 1984. A direct evidence for defect in glucose6-phosphate transport system in hepatic microsomal membrane of glycogen storage disease Type Ib. Biochem. Biophys. Res. Commun. 119: 593-597.

Igarashi, Y., Otomo, H., Narisawa, K., and Tada, K. 1979. A new variant of glycogen storage disease Type I: Probably due to a defect in the glucose-6-phosphate transport system. $J$. Inherit. Metab. Dis. 2: 45-49.

Kamath, S.A., Kummerow, F.A., and Narayan, K.A. 1971. A simple procedure for the isolation of rat liver microsomes. FEBS Lett. 17: 90-92.

Lange, A.J., Arion, W.J., and Beaudet, A.L. 1980. Type Ib glycogen storage disease is caused by a defect in the glucose-6-phosphate translocase of the microsomal glucose-6-phosphatase system. J. Biol. Chem. 255: 8381-8384.

Narisawa, K., Igarashi, Y., Otomo, H., and Tada, K. 1978. A new variant of glycogen storage disease Type I: Probably due to a defect in the glucose-6-phosphate transport system. Biochem. Biophys. Res. Commun. 83: 1360-1364.

Narisawa, K., Otomo, H., Igarashi, Y., Arai, N., Otake, M., Tada, K., and Kuzuya, T. 1983. Glycogen storage disease Type Ib: Microsomal glucose-6-phosphatase system in two patients with 
different clinical findings. Pediatr. Res. 17 : 545-549.

Narisawa, K., Tada, K., and Kuzuya, T. 1981. Neutropenia in type Ib glycogen storage disease. J. Pediatr. 99: 334-335.

Nordie, R.C. and Arion, S.J. 1965. Liver microsomal glucose-6-phosphatase, inorganic pyrophosphatase and pyrophosphate-glucose phosphotransferase. J. Biol. Chem. 240: 2155-2164.

Nordie, R.C. and Arion, W.J. 1966. Glucose-6-phosphatase. In Methods in Enzymology, IX, Wood et al., eds. Academic Press, New York, pp. 619-625.

Schaub, J., Bartholome, K., Feist, D., and Schmidt, H. 1981. Glycogenosis type Ib: Further evidence for a membrane disease. Eur. J. Pediatr., 135: 325.

Tada, K., Narisawa, K., and Igarashi, Y. 1983. Genetic heterogeneity of glycogen storage disease Type Ib. J. Inherit. Metab. Dis. 6: 129-130. 